processes (Pointers for Service Change) that warrant consideration for those aiming to improve the experience of care in hospital for people living with dementia, their carers and staff.

An advisory group of dementia specialists, hospital staff, commissioners and family carers advised us throughout the project. PROSPERO registration CRD42018086013.

Results Our reviews show that the experience of care in hospital for people living with dementia is a dynamic process, being impacted at any one time by a complex range of personal, institutional and environmental factors. Despite the intent to deliver person centered care, and armed with the knowledge of how important this is particularly for people living with dementia, this is still not happening consistently across hospital care. The effect of this is not only a poorer experience of care for people living with dementia and their carers, but also has a detrimental effect on staff emotional well-being as a result of not being able to give the care they strive to provide.

Working closely with evidence end-users enabled us to transform these findings into easily-accessible, practical suggestions to improve the experience of care: Dementia Understanding, Education and Training, Modelling Person Centred Care, Environment, Not Alone, Time, Information Sharing, Access to Resources, Communication, Ask Family, Raise the Profile and Engage Volunteers.

Conclusion Evidence suggests that although people living with dementia can have a good experience of care in hospital, this is still not happening for many. When staff cannot provide the care they would like to give, this has a negative effect on people living with dementia, their carers and the staff themselves. Future research should identify how best to change ward cultures, and how to maintain these changes in the long-term.

\section{P03 ALCOHOL CONSUMPTION AND COGNITIVE FUNCTION IN OLDER POPULATION IN CHINA}

${ }^{1} \mathrm{AY} \mathrm{Bai}^{*},{ }^{2} \mathrm{JS}$ Chen, ${ }^{3} \mathrm{BM} \mathrm{Fu},{ }^{4} \mathrm{H}$ Bo. ${ }^{1}$ Public Health, Peking University, Beijing, China; ${ }^{2}$ Life Sciences, Peking University, Beijing, China; ${ }^{3}$ Mathematical Sciences, Peking University, Beijing, China; ${ }^{4}$ Bradford Institute for Health Research, Bradford Teaching Hospitals NHS Foundation Trust, Bradford, UK

\subsection{6/jech-2020-SSMabstracts.99}

Background With economic development and associated multidimensional social changes, the past three decades have witnessed striking increase in alcohol consumption in China compared to other countries. Cognitive impairment is a serious threat to the health of the elderly in the context of demographic aging process. Previous cross-sectional studies and clinical trials have documented inconsistent conclusions on whether drinking alcohol has dose-response association with cognitive function in the literature. Using a nationally representative and longitudinal dataset, this research intends to examine the relationship between alcohol consumption and cognitive performance in elderly population in China.

Methods We used 2011-2013 longitudinal data from the China Health and Retirement Longitudinal Study (CHARLS) comprising 17314 participants with an average age of 59 years. Alcohol consumption was measured by drinking status (never, former, moderate, excessive drinkers) based on number of standard drinks per week. We studied mental intactness and episodic memory function as measures of cognitive functioning. Lagged dependent variable models were used to examine independent associations between alcohol consumption and cognitive functioning. Our models controlled for demographic factors, socioeconomic factors, baseline cognitive functioning and indicator for lifestyle. We also tested for an inverted J shaped relationship between drinking alcohol and cognitive functioning.

Results A total of 10404 nondrinkers (60.09\%), 2450 former drinkers (14.15\%), 1599 moderate drinkers (9.24\%) and 1525 excessive drinkers $(8.81 \%)$ were included. Compared to never drinkers, there were no statistically significant associations between this group and moderate drinking group. While, excessive drinkers were consistently associated with on average 0.13 -point decrease in episodic memory scores fully adjusted model $(\mathrm{P}=0.031)$. For mental intactness, there were no statistically significant differences between never drinkers and other groups. Furthermore, we did not find evidence to support a J-shaped association between standard drinks per week and measures of cognitive functioning.

Conclusion Excessively drinking alcohol was associated with greater decline in episodic memory function, but not mental intactness. There is no association between moderate drinking group and cognitive functioning measures in elder Chinese population.

\section{P04 CHILDHOOD GROWTH AND DEVELOPMENT AND DNA METHYLATION AGE IN MID-LIFE}

1J Maddock* ${ }^{*}{ }^{2} \mathrm{~J}$ Castillo-Fernandez, ${ }^{1} \mathrm{~A}$ Wong, ${ }^{3} \mathrm{KK}$ Ong, ${ }^{4} \mathrm{~GB}$ Ploubidis, ${ }^{4} \mathrm{~A}$ Goodman, ${ }^{1} \mathrm{D}$ Kuh, ${ }^{2} \mathrm{~B}$ Bell, ${ }^{5} \mathrm{R}$ Hardy. ${ }^{1} \mathrm{M} R \mathrm{C}$ Unit for Lifelong Health and Ageing, University College London, London, UK; ${ }^{2}$ Department of Twin Research and Genetic Epidemiology, King's College London, London, UK; ${ }^{3}$ MRC Epidemiology Unit and Department of Paediatrics, University of Cambridge School of Clinical Medicine, Cambridge, UK; ${ }^{4}$ Centre for Longitudinal Studies, University College London, London, UK; ${ }^{5}$ CLOSER, University College London, London, UK

10.1136/jech-2020-SSMabstracts. 100

Background Biomarkers of ageing based on DNA methylation (DNAm) have recently been developed. The first (Hannum and Horvath) and second generation (Levine and GrimAge) DNAm biomarkers predict survival, age-related disease and functional capabilities better than chronological age alone; with second generation biomarkers showing more consistent and stronger associations. In the first study of its kind, we examine the association between growth in early life, pubertal timing and DNAm age biomarkers in mid-life.

Methods Participants from the Medical Research Council (MRC) National Survey of Health and Development who had information on DNAm were analysed (NSHD, $n=1,376$ ). Four DNAm age acceleration (AgeAccel) biomarkers were calculated when participants were aged 53 years: AgeAccelHannum, AgeAccelHorvath, AgeAccelLevine and AgeAccelGrim. Birthweight and weight and height across infancy, childhood and adolescence were measured. Pubertal timing was established using age at menarche (girls) and pubertal stage at 1415 years (boys). The relationship between weight and height change in infancy (2-4), childhood (4-7) and adolescence (715 ) and AgeAccel was investigated using regression with conditional growth measures; sex-specific residuals from a regression of current size on previous size. Linear regression models examined associations between pubertal timing and AgeAccel. We replicated analyses using height and weight from late childhood ( $7 \mathrm{y})$ to adolescence (16 y) and pubertal timing among 240 participants at 45 years from The National Child and Development Study (NCDS). 
Results A one standard deviation (SD) increase in weight gain between 7 and 15 years was associated with 0.50 years $(95 \%$ CI: $0.21,0.80$ ) higher AgeAccelGrim and, with 0.22 years (95\% CI: -0.11, 0.55) higher AgeAccelLevine. This was replicated in NCDS. For linear growth, there was some evidence that more rapid growth between 2 and 4 years was associated with lower AgeAccelLevine (-0.39 years [95\% CI: -0.74, $0.050)$ and AgeAccelGrim (-0.24 years [95\% CI: $-0.54,0.06])$. There was no evidence that relative weight gain and linear growth during childhood was associated with any other AgeAccel biomarker. There was no relationship between pubertal timing in men and any of the AgeAccel biomarkers at 53 years. Women who reached menarche $\geq 12$ years had 1.20 years $(95 \%$ CI: $0.15,2.24)$ higher AgeAccelGrim on average than women who reached menarche $<12$ years; however this was not replicated in NCDS.

Conclusion Our findings support the use of the second generation DNAm age biomarkers as markers of ageing and reinforces the idea that faster gains in weight during adolescence has lasting implications for healthy ageing.

\section{P05 PERFORMANCE ON DIFFERENT COGNITIVE TESTS PREDICT FUTURE DEMENTIA: FIFTEEN YEARS OF FOLLOW-UP IN A BRITISH COHORT STUDY}

SA Hayat*, R Luben, KT Khaw, C Brayne. Public Health and Primary Care, University of Cambridge, Cambridge, UK

\subsection{6/jech-2020-SSMabstracts. 101}

Background Current policies do not support screening for future dementia among apparently healthy individuals. This is due to the lack of certainty of the clinical outcome and effective interventions. Numerous dementia risk models have been developed, currently, all within the research setting only. Studies have shown impairment in multiple cognitive domains several years before a clinical diagnosis of dementia.

Methods We examined the utility of an extensive battery assessing a range of specific cognitive abilities as well as a composite global score, to predict dementia. Dementia outcomes were ascertained using electronic health record linkage in 8581 individuals (aged 48-92 years) taking part in the EPIC-Norfolk study. Participants were followed for 15 years (2004-2019). Risk of dementia was estimated using Cox proportional hazard models adjusting for sociodemographic, lifestyle and health variables, evaluating discriminative accuracy of the models by analysing receiver operating characteristic (ROC) curves.

Results Poor cognition was predictive of incident dementia, even after adjustment for co-variates. Those with a poor performance score for global cognition (bottom 10\%) were almost four times as likely to get a dementia diagnosis than those who performed well $(\mathrm{HR}=3.51$ (95\%CI 2.61, 4.71) $\mathrm{P}<0.001)$. Associations were observed for specific as well as global cognitive abilities. The test for episodic (verbal) memory outperformed other tests and was comparable to global cognition scores. Poor cognition in four or more tests was associated with 10 -fold increased risk of developing dementia compared to those not performing poorly in any test $(\mathrm{HR}=10.82(95 \%$ CI $6.85,17.10) \mathrm{P}<0.001))$. Cognitive measures strengthen prediction models of dementia (AUC $=0.85$ (95\%CI 0.82, 0.87) $\mathrm{P}<0.001)$.

Discussion This study provides further insight on poor cognition predicting future dementia. This association was observed for global cognition and specific abilities, particularly for verbal episodic memory. Impairment occurs in multiple domains several years prior to a clinical diagnosis, and the more pervasive and greater the variability, the higher the risk of dementia. Deficits across multiple domains predict over and above individual test scores.

\section{P06 SCHIZOPHRENIA POLYGENIC RISK PREDICTS GENERAL COGNITIVE DEFICIT BUT NOT FURTHER COGNITIVE DECLINE IN HEALTHY OLDER ADULTS}

1,20 Ainakina*, ${ }^{3}$ A Kẹpińska, ${ }^{3} \mathrm{~J}$ MacCabe, ${ }^{1} \mathrm{D}$ Cadar, ${ }^{1} \mathrm{~A}$ Steptoe, ${ }^{3} \mathrm{R}$ Murray. ${ }^{1}$ Department of Behavioural Science and Health, Institute of Epidemiology and Health Care, University College London, London, UK; ${ }^{2}$ Department of Biostatistics and Health Informatics, Institute of Psychiatry, Psychology and Neuroscience, King's College London, University of London, London, UK; ${ }^{3}$ Department of Psychosis Studies, Institute of Psychiatry, Psychology and Neuroscience, King's College London, London, UK

\subsection{6/jech-2020-SSMabstracts. 102}

Background There has been a long argument over whether or not schizophrenia is a neurodegenerative disorder associated with progressive brain changes and increasing cognitive impairment. Building on the fact that schizophrenia is highly heritable and overlaps genetically with general cognitive ability, we investigated whether common genetic variants associated with schizophrenia additively confer a stable deficit in cognitive ability, a greater risk of cognitive decline over time, or both, over the 8-year follow-up, independently from the effects of the apolipoprotein $\mathrm{E}$ gene (APOE- $\epsilon 4$ ), in phenotypically welldefined sample of healthy older adults.

Methods We used data from the English Longitudinal Study of Ageing study which encompassed 6817 population-representative English adults who were followed-up for 8 years. Cognitive function was measured with well-established tests of memory (tests of immediate and delayed word recall combined into a single measure of correctly recalled words) and executive function (a test of verbal fluency where participants name as many animals as possible in a minute). Polygenic score for schizophrenia (SZ-PGS) was calculated based on the results from Psychiatric Genomics Consortium. Linear mixed effect models with maximum likelihood estimation were used to estimate baseline status and rate of change in cognition associated with SZ-PGS.

Results The sample baseline mean age was 64.3 years old (standard deviation $(\mathrm{SD})=9.3, \quad$ range $=50-101) ; \quad 25.3 \%$ $(\mathrm{N}=1724)$ of participants were carriers of APOE- $\epsilon 4$ and $46.2 \%(\mathrm{~N}=3159)$ were men. The average baseline memory score was $10.4(\mathrm{SD}=3.4)$ and executive function score was 20.7 (SD=6.3). One standard deviation increase in SZ-PGS was associated with a lower baseline executive function score $(-0.23$, 95\%CI-0.38 - -0.08) but not memory. SZ-PGS was not associated with rates of change in these cognitive domains during the 8-year follow-up period. However, APOE- $\epsilon 4$, tobacco smoking and lower wealth were associated with a decrease in the rate of memory and executive function during follow-up.

Discussion Common genetic variants associated with schizophrenia additively confer a stable deficit in cognitive ability but not cognitive ageing. The fact that we observed cognitive decline in our sample over the 8-year follow-up, associated with of APOE- $\epsilon 4$, tobacco smoking and lower wealth demonstrates that our study had the capacity to show cognitive 